\title{
Inflammation-associated lymphangiogenesis: a double-edged sword?
}

\author{
Honsoul Kim,, ${ }^{1,2}$ Raghu P. Kataru,3,4 and Gou Young Koh ${ }^{3,4}$
}

\begin{abstract}
1Department of Radiology and ${ }^{2}$ Research Institute of Radiological Science, Severance Hospital, Yonsei University College of Medicine, Seoul, Republic of Korea. ${ }^{3}$ Laboratory of Vascular Biology and Stem Cells and ${ }^{4}$ Graduate School of Medical Science and Engineering, Korea Advanced Institute of Science and Technology, Daejeon, Republic of Korea.
\end{abstract}

\begin{abstract}
Lymphangiogenesis and lymphatic vessel remodeling are complex biological processes frequently observed during inflammation. Accumulating evidence indicates that inflammation-associated lymphangiogenesis (IAL) is not merely an endpoint event, but actually a phenomenon actively involved in the pathophysiology of various inflammatory disorders. The VEGF-C/VEGFR-3 and VEGF-A/VEGF-R2 signaling pathways are two of the best-studied pathways in IAL. Methods targeting these molecules, such as prolymphangiogenic or antilymphatic treatments, were found to be beneficial in various preclinical and/or clinical studies. This Review focuses on the most recent achievements in the fields of lymphatic biology relevant to inflammatory conditions. Additionally, preclinical and clinical therapies that modulate IAL are summarized.
\end{abstract}

\section{Introduction}

Inflammation is a complex biological reaction associated with the protection of tissues against harmful agents such as microbes, damaged cells, or irritants. The inflammation process is known to accompany several localized and/or systemic events $(1,2)$, such as vascular responses, migration and activation of leukocytes, and systemic inflammatory response syndrome. Additionally, lymphangiogenesis is frequently encountered during inflammation (3-5).

To relieve the hostile microenvironment causing inflammation, noxious antigens and debris should be immunologically removed. In parallel, the excessive interstitial fluid and edema should be drained. In this sense, under inflammatory conditions, the demand for efficient lymphatic drainage increases. Accumulating evidence suggests inflammation-associated lymphangiogenesis (IAL) is not merely an endpoint phenotype of inflammation but rather a dynamic, context-dependent reaction that can alter the natural course of the inflammatory process and/or tissue repair $(3,6,7)$. IAL can profoundly influence the symptomatic presentation of inflammation; for example, lymphatic vessels play a role in regulating tissue fluid clearance $(8,9)$ and macrophage/DC recruitment $(10,11)$. Consequently, the course of IAL may directly influence the degree of mucosal edema and/or leukocyte infiltration, which may partially explain why cardinal signs of inflammation, namely redness, pain, heat, swelling, and loss of function $(1,2)$, appear at inflamed tissues. Recently, several prolymphangiogenic and antilymphatic therapies have been developed, supporting the notion that lymphatic vessels and IAL are potential therapeutic targets that can be exploited to achieve symptomatic control and an improved prognosis $(5,12)$.

Despite advances in our understanding of IAL, there are many concerns with clinical and preclinical lymphatic modulation. The lymphatic system drains directly into the systemic circulation and depends on the LNs to filter and remove debris and pathogens contained in the lymphatic fluid (13). If lymph-borne pathogens can bypass the surveillance of the draining LNs, they can hijack the lymphatic system to gain access to the systemic circulation (14). Consequently, therapeutic approaches designed to promote lymphatic

Conflict of interest: The authors have declared that no conflict of interest exists. Citation for this article: J Clin Invest. 2014;124(3):936-942. doi:10.1172/JCI71607. drainage have the potential risk of saturating nodal surveillance, increasing systemic exposure to unfiltered pathogens and inflammatory mediators. Therefore, lymphatic-modulating therapies could turn out to be "a road to hell paved with good intentions". These therapies should aim not only to improve symptoms, but also to correct the underlying pathogenesis of the target disease. To achieve this goal, it is necessary to expand our understanding of lymphatic biology at the bench and to carefully apply this knowledge in the form of treatments at the bedside. This Review discusses studies from the perspective that the lymphatic system is closely linked with the symptomatic presentation and pathophysiology of certain inflammatory conditions. In addition, recent advances in preclinical and clinical treatments of IAL, which aim to control the natural course of inflammatory disorders, are summarized.

\section{IAL}

Lymphangiogenesis takes place under certain pathological situations, including inflammation and tissue repair (7). Upon initiation of inflammation, the lymphatic system is activated and both the extranodal lymphatic vessels at peripheral tissues and the intranodal lymphatic vessels display exuberant growth and vigorously expand in response to inflammatory stimuli (5). Previously, postnatal lymphangiogenesis was believed to take place exclusively through local sprouting of the preexisting lymphatic endothelial cells (LECs) (lymphangiogenesis; Figure 1). Although this remains the mainstream theory, some recent evidence suggests that circulating progenitor cells might be incorporated directly into the growing lymphatic vessels and transdifferentiate into LECs (lymphovasculogenesis; Figure 1 and refs. 15-20). Macrophage transdifferentiation was reported in an experimental orchitis model (15), LPS-induced peritonitis model (19), corneal injury model (16), and in a human transplanted kidney (17). Interestingly, under normal conditions the lymphatic endothelial progenitor cells appear only in minute quantities, but inflammatory stimuli lead to remarkable increases (18). It is notable that all of these observations supporting the presence of lymphatic endothelial progenitor cells and lymphovasculogenesis have been made under inflammatory conditions (16-19). Nevertheless, the concepts of lymphovasculogenesis and macrophage transdifferentiation remain unclear and require further examination. 


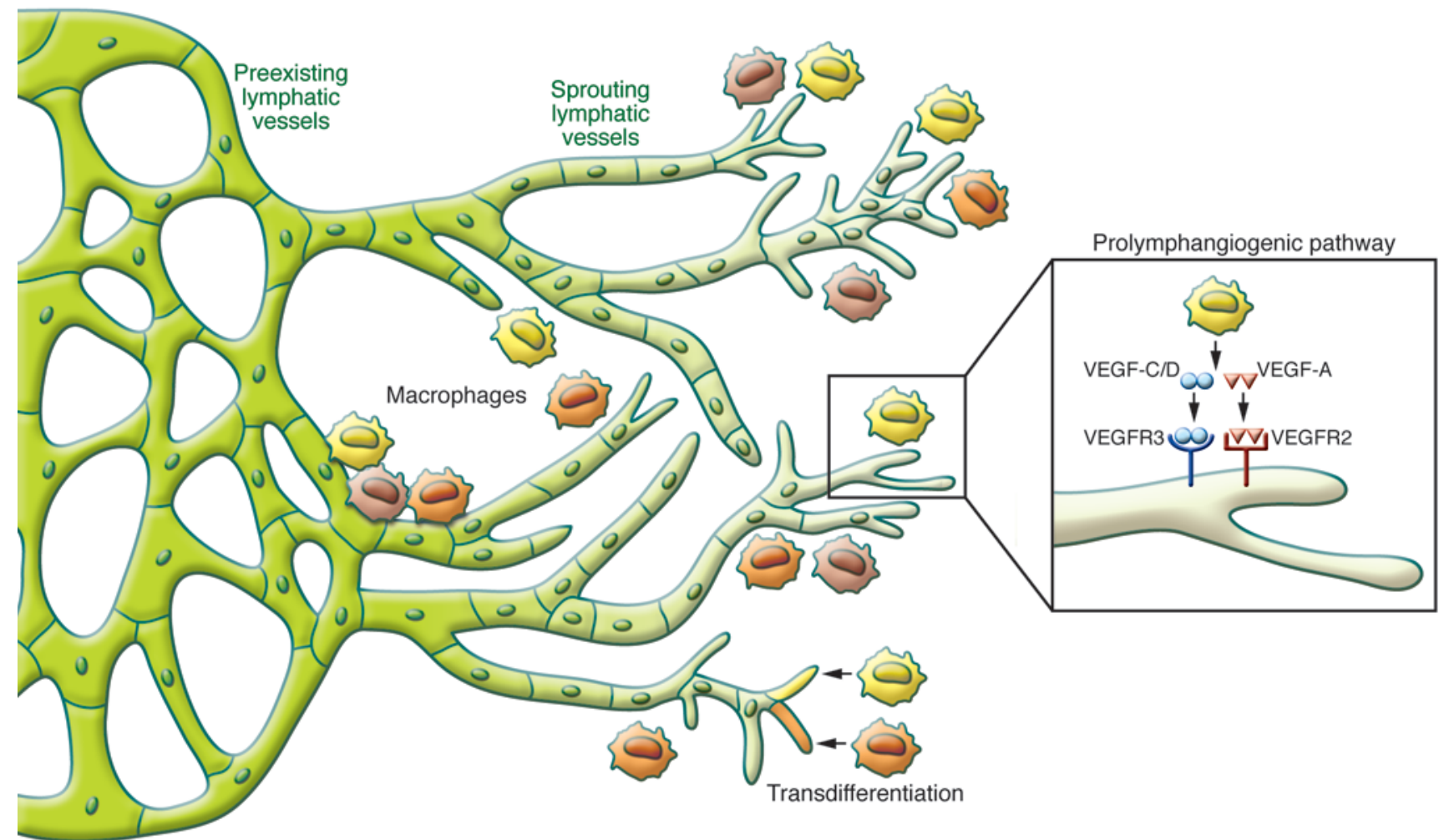

Figure 1

Schematic diagram depicting IAL observed during the acute phase of inflammation. Upon activation by inflammatory stimuli, a local sprouting at the preexisting lymphatic vessels actively occurs in response to macrophage-secreted VEGF. As a result the lymphatic network grows and expands. During this process the VEGF-C/VEGFR-3 and VEGF-A/VEGF-R2 signaling pathways are known to have profound importance, yet other signaling pathways also seem to be involved. It has been suggested that lymphangiogenic macrophages can become incorporated into the lymphatic vessels and transdifferentiate into LECs, but this hypothesis requires more validation.

\section{Signaling pathways involved in IAL}

Many prolymphangiogenic signaling pathways are activated upon exposure to inflammatory stimuli, while the role of VEGF-C/ VEGFR-3 and VEGF-A/VEGF-R2 are the best characterized in the process of IAL $(4,5)$. Transgenic overexpression of VEGF-C resulted in an increase and enlargement of subcutaneous lymphatic vessels. Interestingly, VEGF-C-associated increases in lymphanogenesis relieved the severity of acute skin inflammation and edema observed in oxazolone-induced delayed type hypersensitivity reactions and ultraviolet B irradiation models (21). In contrast, other studies showed that inhibition of the VEGF-C/VEGFR-3 axis suppresses lymphangiogenesis (22-24). In addition, VEGFR-3 inhibition was found to relieve the severity of inflammatory symptoms in rheumatoid arthritis and LPS-induced acute inflammation models $(23,25)$.

VEGF-A is involved in another signaling pathway that induces prolymphangiogenic effects in a context-dependent manner. It is debatable whether the action and effect of VEGF-A on IAL is a direct or indirect consequence of this signaling pathway (23). VEGF-A also induces lymphatic vessel remodeling, as mice overexpressing VEGF-A have been shown to display enlarged lymphatic vessels (26). Interestingly, exposure to chronic cutaneous inflammation induced lymphangiogenesis and lymphatic vessel hyperplasia in VEGF-A overexpressing mice, but not in wild-type mice (26). In contrast, inflammation was efficiently suppressed by simultaneous inhibition of VEGF-R1 and VEGF-R2 (26).
Other signaling pathways, such as those involving TNF- $\alpha$ (23, 27), lymphotoxin- $\alpha$ (28), toll-like receptor signaling (29), NF-кB (30), erythropoietin (31), COX-2, and prostaglandin E2 receptor signaling $(32,33)$ also influence prolymphangiogenesis and are closely involved in the process of IAL.

Antilymphatic signaling pathways repress IAL. IFN- $\gamma$ modulates the JAK/STAT pathway and has a strong antilymphatic effect. It is particularly important for post-inflammatory regression of the already expanded neo-lymphatic network within the regional LN (34). TGF- $\beta 1$ (35-37), endostatin (38), and thrombospondin (39) also have an antilymphatic role (40). However, our knowledge of the negative regulatory mechanisms of IAL remains relatively limited, and further studies are necessary to identify other antilymphatic signaling pathways.

\section{The balance between prolymphangiogenic and antilymphatic elements determines the actual state of IAL}

Intranodal follicular B cells (41, 42), CD11b macrophages (25, 43), and fibroblast-type reticular stromal cells (44) are well recognized sources of prolymphangiogenic molecules including VEGF-A and VEGF-C, while T lymphocytes are the main source of the antilymphatic IFN- $\gamma$ (34). The actual amplitude and pattern of IAL is determined by the balance of prolymphangiogenic and antilymphatic elements, which leads to a bi-directional regulatory 


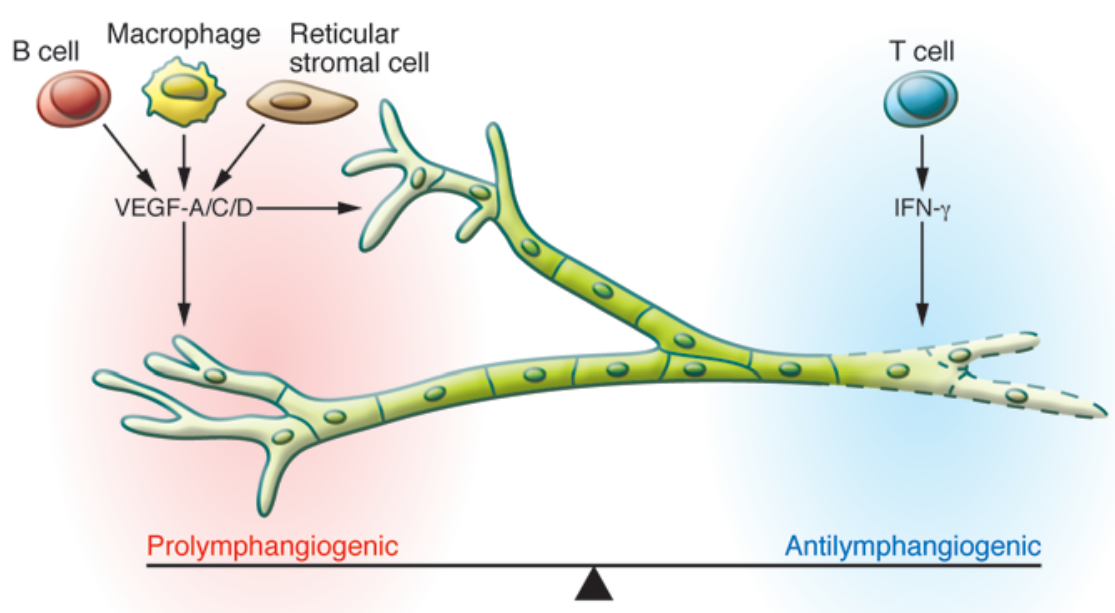

\begin{abstract}
Figure 2
In LNs, the balance between the prolymphangiogenic factors (VEGF-A, VEGF-C, VEGF-D) and antilymphangiogenic factors (IFN- $\gamma$ ) influences the actual response of lymphatic vessels to inflammatory stimuli; accordingly, lymphatic vessels either grow or regress. During the acute stage of inflammation, the prolymphangiogenic factors usually dominate, in which case the final outcome is growth of the lymphatic vessels. As inflammation resolves, the antilymphangiogenic effect increases. Other regulators with either prolymphangiogenic or antilymphangiogenic effects have been reported; however, further studies are necessary before their role can be generalized. The coordination between stromal cells, immune cells, and interstitial flow seem to be other factors that affect the spatiotemporal regulation of IAL.
\end{abstract}

system (Figure 2 and refs. 5, 34, 40). This allows considerable flexibility and accounts for how the lymphatic system can be context dependent and dynamically respond to its microenvironment. For example, skin exposure to either LPS or the T cell mitogen concanavalin-A induces similar inflammatory phenotypes; however, only LPS induces significant IAL (34).

The dynamic phenotypic changes observed during IAL may be explained by the spatiotemporal balance between prolymphangiogenic and antilymphangiogenic drives. Peripheral tissue inflammation induces robust lymphangiogenesis in the draining LNs $(34,45,46)$. During the early phase of inflammation, the prolymphangiogenic factors increase and initiate proliferation of the nodal subcapsular lymphatic vessels. At the peak of inflammation, the prolymphangiogenic factors completely dominate the antilymphatic factors; thus, the lymphatic network spreads out. As inflammation resolves, the balance moves toward the antilymphatic factors and the expanded neo-lymphatic network regresses $(25,34,47)$. In parallel, the coordination between stromal cells, immune cells, and interstitial flow affects the intranodal distribution of VEGF-A to influence the spatiotemporal pattern of IAL (48). Meanwhile, extranodal IAL at the peripheral tissues follows a different natural course, in which the bulk of expanded extranodal lymphatic vessels tend to remain after inflammation resolves $(22,49)$. This may reflect the absence of strong antilymphatic factors, such as intranodal $\mathrm{T}$ cells and IFN- $\gamma$, which would induce lymphatic regression in the peripheral tissue after inflammation is resolved.

Recently, Kelley et al. proposed the concept of lymphatic vessel memory (50). Upon recurrent inflammation, lymphatic vessel memory provokes an accelerated expansion of structurally distinct but functioning lymphatic vessels. Of note, this category of lymphangiogenesis depends on neither the VEGF-C/VEGFR-3 axis nor the VEGF-A/VEGF-R2 axis (50). Further study is necessary to elucidate how this novel mechanism contributes to IAL.

\section{Lymphatic vessels and immune cells closely interact during inflammation}

LECs are directly and indirectly involved in the regulation of tolerance and immunity (51). The lymphatic vessels can be considered the "afferent arm" of an immune reflex arc, while the blood vessels serve as the "efferent arm" (52). Under inflammatory conditions, large quantities of immune cells are transported through blood vessels and gather at the inflamed peripheral tissue. Lymphatic vessels locally promote the recruitment of macrophages and DCs that have gathered at the peripheral tissue. Antigen-bearing DCs and macrophages enter the lymphatic vessels at peripheral tissue through discontinuous endothelial junctions or preformed portals $(53,54)$ and continue to travel into regional LNs. The recruited immune cells further provoke IAL by secreting prolymphangiogenic factors $(10,11)$. Such reciprocal interactions between lymphatic vessels and immune cells contribute to the immune system's response to inflammatory stimuli.

Macrophages are one of the most important types of immune cells during IAL. Bone marrow-derived myeloid cells/macrophages are dispatched into the circulation and are transported via blood vessels to the inflamed tissue, where they phagocytose pathogens and clear debris. They then move from the peripheral tissue to regional LNs through lymphatic vessels, which is an important process for resolving inflammation (55). LECs express chemotactic molecules that promote macrophage infiltration. In turn, macrophages secrete paracrine prolymphangiogenic growth factors such as VEGF-C, VEGF-D, and VEGF-A in response to inflammatory stimuli $(20,56,57)$. Increased levels of VEGF-A enhance angiogenesis, which acts to reinforce immune cell recruitment (23). These interactions between macrophages and lymphatic vessels lead to a reciprocal regulatory relationship in the process of IAL. Another important role of macrophages during IAL is transdifferentiation into LECs (15-19), as described above.

DCs have a major role in adaptive immunity, as they serve as APCs; therefore, proper recruitment and migration of DCs are critical to the inflammatory response. LECs aid the recruitment of DCs by producing chemokines that are sensed by hematopoietic cells (for example, LECs express CCL21, which recruits CCR7 ${ }^{+}$DCs) $(10,11,58,59)$. Inflammatory cytokines provoke both LECs and DCs to recruit more DCs. Inflammatory stimuli trigger LECs to express leukocyte adhesion receptors, such as ICAM-1, VCAM-1, and E-selectin, which mediate trafficking of DCs through the afferent lymphatic vessels (60). Meanwhile, inflammatory cytokines promote DC maturation and their migratory capacity (61).

Studies $(62,63)$ on solid organ transplantation revealed that VEGFR-3 signaling is also involved in mediating migration of DCs toward the draining LN. In a rat cardiac allograft model, 
interfering with VEGFR-3 signaling inhibited LEC production of CCL21, an important chemokine required to accomplish the homing of activated CCR7 APCs to secondary lymphoid tissue. Consequently, modulating the activities of VEGFR-3 signaling may influence adaptive immunity (63). Similarly, in a corneal transplantation model, local suppression of VEGFR-3 inhibited graft origin DC migration to the recipient draining $\mathrm{LN}$ (62). This reduced donor-specific delayed hypersensitivity and improved graft survival. In this context, reciprocal interactions between the lymphatic vessels and immune cells in response to inflammatory stimuli during IAL can ultimately affect the course of adaptive immunity.

\section{Lymphatic vessels and respiratory tract inflammation}

Under normal conditions, lymphatic vessels of the respiratory tract adopt a microstructure optimized to facilitate the entrance of immune cells and interstitial fluid. The basement membrane of lymphatic vessels is discontinuous, especially at the initial lymphatic capillaries, through which inflammatory cells frequently pass. Unlike the continuous zipper-like junctions (zippers) of endothelial cells that effectively seal up the borders of collecting lymphatic channels and blood vessels, initial lymphatics display LECs with overlapping flaps anchored simply by discontinuous, button-like junctions (buttons) $(53,54)$. Interestingly, 14 days after infection with Mycoplasma pulmonis, the airway presented with growing lymphatic sprouts, which had zippers instead of buttons (53). Such remodeling of the lymphatic vessels makes them less permeable and contributes to impaired fluid clearance and mucosal edema (8). Prophylactic administration of dexamethasone in the same airway inflammation model was found to largely prevent remodeling of both blood vessels and lymphatic vessels; however, once the inflammation resolved, it was less effective for treating the mice. Dexamethasone treatment reversed most of the blood vessel remodeling caused by inflammation, but it failed to reverse the lymphangiogenesis (49), although some microstructural alterations, such as button formation, were observed (8). Antilymphatic treatment with anti-VEGFR-3 antibody (mF4-31C1) inhibited additional lymphangiogenesis but failed to suppress lymphatic vessels that had already been induced before the start of treatment. After inflammation resolved, the number of the sprouts decreased and their microstructure once again consisted of buttons (53).

Studies on human idiopathic pulmonary fibrosis, which accompanies chronic inflammation and ultimately ends in pulmonary fibrosis and respiratory failure, suggested newly formed lymphatic vessels are probably part of the pathophysiology and contribute to the fibrotic process and maintenance of injury (64). Lymphatic vessels were observed throughout fibrotic tissue and at the periphery of fibroblastic foci (64). With worsening of the disease, the number of lymphatic vessels did not change, but the newly formed lymphatics displayed morphologic alterations. Bronchoalveolar lavage fluid analysis of patients with idiopathic pulmonary fibrosis demonstrated elevated levels of CCL21 chemokines, which were likely produced by LECs (64). CCL21 can promote fibroblast proliferation by stimulation of its receptor CCR7 and also enhance the recruitment of DCs (64-66). Collectively, chronic inflammatory diseases involving the respiration system may be accompanied by significant structural alterations of lymphatic vessels, which probably are irreversible changes that are resistant to lymphatic modulating treatments.

\section{Inflammatory bowel disease}

Mice deficient for angiopoietin-2 have intestinal lymphatic dysplasia and exacerbated colitis in the dextran sulfate sodium colitis model $(67,68)$, suggesting that lymphatic vessels are functionally important for the resolution of bowel inflammation. Selective ablation of the lymphatic vessels led to distortion of the intestinal villi architecture and severe bowel inflammation (69). In a human study of ulcerative colitis, the disorder of the lymphatic vessel architecture reflected disease activity (70). These observations imply that the integrity of intestinal lymphatic vessels is essential to maintain the structure and function of intestinal villi. Similarly, antilymphatic treatment with anti-VEGFR-3 antibodies in an animal model of inflammatory bowel disease aggravated inflammation and submucosal edema, increased leukocyte infiltration, and caused the lymphatic vessels to become enlarged and tortuous (67). Although the precise pathophysiology of inflammatory bowel disease is unknown, lymphatic vessel obstruction and dysfunction are long-recognized features observed in humans with this disease (71). Lymphangiectasia and lymphocytic perilymphangitis were also found in patients with Crohn's disease, suggesting that lymphatic derangements are a part of the pathogenesis (72, 73). The presence of lymphangiogenesis is another frequently reported feature in both Crohn's disease and ulcerative colitis $(70,74,75)$. Among patients with Crohn's disease, relatively lower lymphatic vessel density was associated with a higher risk of endoscopic disease recurrence after surgery, suggesting that patients could benefit from improved lymphatic flow (76). However, it remains controversial whether lymphatic expansion promotes bowel inflammation and injury or represents a protective or adaptive response. Disturbances in lymphatic function seem to correspond with aggravation of inflammatory bowel disease; therefore, lymphangiogenic remodeling could have benefits, at least in the early phases of disease $(76,77)$, but further research on this issue is necessary.

\section{Diabetes}

Accumulating evidence indicates that inflammation is an important contributor to insulin resistance. Chronic inflammatory elements, such as macrophage-derived TNF- $\alpha$, promote type 2 diabetes $(78,79)$. In a diabetic animal model, lymphangiogenesis occurred around islet cells and contributed to the pathogenesis of inflammation. Suppression of VEGFR-3 reduced lymphangiogenesis at islets and draining LNs; consequently, inflammation decreased, while islets were preserved (80). Moreover, functional alteration of the lymphatic system was also observed in a diabetic rat model (81). In patients with type 2 diabetes, the dermal lymphatic system showed increased lymphatic vessel density and proliferation. Freshly isolated human dermal LECs from these patients exhibited a distinct gene expression signature, including enhanced expression of chemotactic factors and leukocyte trafficking receptors (CXCL10, VCAM-1, and CMTM7). This resulted in increased recruitment of macrophages, which can serve as a major source of pro-inflammatory cytokines, including TNF- $\alpha$. Consequently, the aberrant lymphatic vessel growth observed in type 2 diabetes could account for the persistent inflammation that impairs wound healing (82). Further studies are necessary to determine whether IAL under diabetic conditions is also associated with other major complications, such as macroangiopathy, microangiopathy, and neuropathy. 


\section{Transplantation and rejection}

Immune-mediated graft rejection is one of the most devastating and challenging events encountered after organ transplantation. There is a great need for strategies to modulate the recipient's immunity to promote graft survival and function while avoiding excessive immunosuppression. Afferent lymphatic vessels serve as a route for the delivery of both donor and recipient APCs, which carry allogenic tissue antigens to the regional LNs (83). Increased lymphangiogenesis promotes the migration of APCs carrying foreign tissue antigens to the recipient's draining LNs, which provokes inflammation and is assumed to facilitate the initial immune cascade events that trigger adverse immune reactions. Some studies have shown that interference with lymphatic function suppresses lymphangiogenesis and prolongs graft survival $(52,83)$. Other studies, using an experimental corneal graft model, identified early post-transplantation lymphangiogenesis as a significant risk factor for immune rejection after keratoplasty, which can be reduced with postoperative anti-angiogenic/antilymphangiogenic treatments (84-86).

Using suture-induced corneal neovascularization and corneal transplantation murine models, Dietrich et al. demonstrated that preexisting lymphangiogenesis induced by inflammation is a critical factor promoting immune rejections of corneal grafts (52). Encouragingly, even if inflammation was present prior to transplantation, preoperative antilymphatic treatment with either anti-VEGFR-3 antibody or a small-molecule agent against the LEC integrin $\alpha 5 \beta 1$ significantly improved graft survival, suggesting that temporary prophylactic antilymphatic treatment before transplantation could be beneficial (52). A case report describes an allogeneic and autologous cultivated limbal stem cell graft transplantation instead of a conventional keratolimbal allograft (because the antigenicity of the graft is thought to decrease during culture, causing lower rates of rejection; ref. 87) in a patient with a history of two failed penetrating keratoplasties (88). Unfortunately, immune rejection still occurred, and this pioneering attempt failed (88). Lymphangiogenesis was observed in the removed graft, which again emphasizes the importance of IAL in transplantation (88). Another important consideration is the ideal timing of transplantation if inflammation has already occurred. Near complete regression of pathologic corneal lymphatic vessels was observed six months after suture-induced corneal lymphangiogenesis was established in mice (89). This implies that the keratoplasty procedure for patients with a history of corneal inflammation should be delayed, if possible, to secure an inflammation-free interval $(86,89)$.
Similar observations have been made in visceral organ transplants. In a diabetic mouse model transplanted with pancreatic islet allografts, lymphangiogenesis occurred in the islet allografts and in draining LNs after transplantation (83). However, antilymphatic treatment using anti-VEGFR-3 antibody, FTY720, or sunitinib inhibited IAL and significantly prolonged graft survival (83). In human renal transplants (90), the lymphatic vessel density increased in grafts. In addition, lymphatic vessels were closely associated with nodular mononuclear infiltrates, which contained chemokine receptor $\mathrm{CCR} 7^{+}$cells that appeared to have been attracted by LECs. These observations suggest that lymphangiogenesis probably contributes to detrimental alloreactive immune responses and the export of the rejection infiltrate (90). Thus, lymphangiogenesis could be exploited as a therapeutic target for modulating immune rejection and tolerance after cornea and visceral organ transplantation.

\section{Conclusion}

Lymphangiogenesis is one of the most frequently observed signs of inflammation. IAL is not only closely related to inflammatory symptoms, but it can significantly influence the course of inflammation. New methods for lymphatic modulation were recently developed, creating novel treatments for inflammatory disorders. However, researchers and clinicians should keep in mind that lymphatic modulating therapies could be a double-edged sword, and must consider the complexity and context of lymphatic biology during treatment.

Although considerable progress has been achieved, the field of IAL remains new and our understanding of lymphatic biology is far from complete. In particular, research elucidating the pathogenic links between IAL and specific diseases is necessary for reasonable clinical decision-making and evidence-based therapeutic approaches for lymphatic modulation. Further research, both preclinical and clinical, is still required to address issues relevant to IAL, such as autoimmune diseases, immunodeficiency, posttransplant concerns, and metabolic syndromes.

\section{Acknowledgments}

This work was supported by grants (R2009-0079390 and 2013M3A9B6046565 to G.Y. Koh) from the National Research Foundation, and was also funded by the Ministry of Science, ICT and Future Planning of Korea.

Address correspondence to: Gou Young Koh, Graduate School of Medical Science and Engineering, KAIST, 373-1, Guseong-dong, Daejeon, 305-701, Republic of Korea. Phone: 82.42.350.2638; Fax: 82.42.350.2610; E-mail: gykoh@kaist.ac.kr.
1. Mouta C, Heroult M. Inflammatory triggers of lymphangiogenesis. Lymphat Res Biol. 2003; 1(3):201-218.

2. Robbins SL, Kumar V, Cotran RS. Robbins And Cotran Pathologic Basis OfDisease. Philadelphia, Pennsylvania, USA: Saunders/Elsevier; 2010.

3. Alitalo K. The lymphatic vasculature in disease. Nat Med. 2011;17(11):1371-1380.

4. Tammela T, Alitalo K. Lymphangiogenesis: Molecular mechanisms and future promise. Cell. 2010;140(4):460-476.

5. Kim H, Kataru RP, Koh GY. Regulation and implications of inflammatory lymphangiogenesis. Trends Immunol. 2012;33(7):350-356.

6. Avraham T, et al. Fibrosis is a key inhibitor of lymphatic regeneration. Plast Reconstr Surg. 2009;124(2):438-450

7. Paupert J, Sounni NE, Noel A. Lymphangiogen- esis in post-natal tissue remodeling: lymphatic endothelial cell connection with its environment. Mol Aspects Med. 2011;32(2):146-158

8. Yao LC, Baluk P Srinivasan RS, Oliver G, McDonald DM. Plasticity of button-like junctions in the endothelium of airway lymphatics in development and inflammation. Am J Pathol. 2012;180(6):2561-2575.

9. Zgraggen S, Ochsenbein AM, Detmar M. An important role of blood and lymphatic vessels in inflammation and allergy. J Allergy (Cairo). 2013;2013:672381.

10. Alvarez D, Vollmann EH, von Andrian UH. Mechanisms and consequences of dendritic cell migration. Immunity. 2008;29(3):325-342.

11. Randolph GJ, Angeli V, Swartz MA. Dendritic-cell trafficking to lymph nodes through lymphatic vessels. Nat Rev Immunol. 2005;5(8):617-628.

12. Mumprecht V, Roudnicky F, Detmar M. Inflam- mation-induced lymph node lymphangiogenesis is reversible. Am J Pathol. 2012;180(3):874-879.

13. Bielenberg DR, D'Amore PA. All vessels are not created equal. Am J Pathol. 2013;182(4):1087-1091.

14. Kastenmuller W, Torabi-Parizi P, Subramanian N, Lammermann T, Germain RN. A spatially-organized multicellular innate immune response in lymph nodes limits systemic pathogen spread. Cell. 2012;150(6):1235-1248.

15. Hirai $S$, et al. Lymphangiogenesis in chronic inflammation in the testis. Andrology. 2013;1(1):147-154.

16. Maruyama K, et al. Inflammation-induced lymphangiogenesis in the cornea arises from CD11b-positive macrophages. J Clin Invest. 2005; 115(9):2363-2372.

17. Kerjaschki D, et al. Lymphatic endothelial progenitor cells contribute to de novo lymphangiogenesis in human renal transplants. Nat Med. 
2006;12(2):230-234.

18. Lee JY, et al. Podoplanin-expressing cells derived from bone marrow play a crucial role in postnatal lymphatic neovascularization. Circulation. 2010;122(14):1413-1425.

19. Hall KL, Volk-Draper LD, Flister MJ, Ran S. New model of macrophage acquisition of the lymphatic endothelial phenotype. PLoS One. 2012;7(3):e31794.

20. Zumsteg A, Christofori G. Myeloid cells and lymphangiogenesis. Cold Spring Harb Perspect Med. 2012;2(6):a006494.

21. Huggenberger R, et al. An important role of lymphatic vessel activation in limiting acute inflammation. Blood. 2011;117(17):4667-4678.

22. Baluk P, et al. Pathogenesis of persistent lymphatic vessel hyperplasia in chronic airway inflammation. J Clin Invest. 2005;115(2):247-257.

23. Guo R, et al. Inhibition of lymphangiogenesis and lymphatic drainage via vascular endothelial growth factor receptor 3 blockade increases the severity of inflammation in a mouse model of chronic inflammatory arthritis. Arthritis Rheum. 2009; 60(9):2666-2676

24. Wirzenius $M$, et al. Distinct vascular endothelial growth factor signals for lymphatic vessel enlargement and sprouting. J Exp Med. 2007; 204(6):1431-1440.

25. Kataru RP, et al. Critical role of CD $11 \mathrm{~b}+$ macrophages and VEGF in inflammatory lymphangiogenesis, antigen clearance, and inflammation resolution. Blood. 2009;113(22):5650-5659.

26. Kunstfeld R, et al. Induction of cutaneous delayedtype hypersensitivity reactions in VEGF-A transgenic mice results in chronic skin inflammation associated with persistent lymphatic hyperplasia. Blood. 2004;104(4):1048-1057.

27. Zhu M, Fu YX. The role of core TNF/LIGHT family members in lymph node homeostasis and remodeling. Immunol Rev. 2011;244(1):75-84.

28. Mounzer RH, et al. Lymphotoxin-alpha contributes to lymphangiogenesis. Blood. 2010;116(12):2173-2182.

29. Babu S, Anuradha R, Kumar NP, George PJ, Kumaraswami V, Nutman TB. Toll-like receptor- and filarial antigen-mediated, mitogen-activated protein kinase- and NF-KB-dependent regulation of angiogenic growth factors in filarial lymphatic pathology. Infect Immun. 2012;80(7):2509-2518.

30. Flister MJ, et al. Inflammation induces lymphangiogenesis through up-regulation of VEGFR-3 mediated by NF-кB and Prox1. Blood. 2010;115(2):418-429.

31. Lee AS, et al. Erythropoietin induces lymph node lymphangiogenesis and lymph node tumor metastasis. Cancer Res. 2011;71(13):4506-4517.

32. Hosono K, et al. Roles of prostaglandin E2-EP3/ EP4 receptor signaling in the enhancement of lymphangiogenesis during fibroblast growth factor-2-induced granulation formation. Arterioscler Thromb Vasc Biol. 2011;31(5):1049-1058.

33. Kashiwagi S, Hosono K, Suzuki T, Takeda A, Uchinuma E, Majima M. Role of COX-2 in lymphangiogenesis and restoration of lymphatic flow in secondary lymphedema. Lab Invest. 2011; 91(9):1314-1325

34. Kataru RP, et al. T lymphocytes negatively regulate lymph node lymphatic vessel formation. Immunity. 2011;34(1):96-107.

35. Oka M, et al. Inhibition of endogenous TGF- $\beta$ signaling enhances lymphangiogenesis. Blood. 2008;111(9):4571-4579.

36. Clavin NW, et al. TGF- $\beta 1$ is a negative regulator of lymphatic regeneration during wound repair. $A m J$ Physiol Heart Circ Physiol. 2008;295(5):H2113-H2127.

37. Avraham T, et al. Blockade of transforming growth factor-beta 1 accelerates lymphatic regeneration during wound repair. Am J Pathol. 2010; 177(6):3202-3214

38. Ou J, et al. Endostatin suppresses colorectal tumor- induced lymphangiogenesis by inhibiting expression of fibronectin extra domain A and integrin $\alpha 9$. J Cell Biochem. 2011;112(8):2106-2114.

39. Cursiefen C, et al. Thrombospondin 1 inhibits inflammatory lymphangiogenesis by CD36 ligation on monocytes. J Exp Med. 2011;208(5):1083-1092.

40. Zampell JC, et al. Lymphatic function is regulated by a coordinated expression of lymphangiogenic and anti-lymphangiogenic cytokines. Am J Physiol Cell Physiol. 2012;302(2):C392-C404.

41. Angeli V, et al. B cell-driven lymphangiogenesis in inflamed lymph nodes enhances dendritic cell mobilization. Immunity. 2006;24(2):203-215

42. Shrestha B, et al. B cell-derived vascular endothelial growth factor A promotes lymphangiogenesis and high endothelial venule expansion in lymph nodes. J Immunol. 2010;184(9):4819-4826.

43. Kim KE, et al. Role of CD11b+ macrophages in intraperitoneal lipopolysaccharide-induced aberrant lymphangiogenesis and lymphatic function in the diaphragm. Am J Pathol. 2009;175(4):1733-1745.

44. Chyou S, et al. Fibroblast-type reticular stromal cells regulate the lymph node vasculature. J Immunol. 2008;181(6):3887-3896.

45. Halin C, Tobler NE, Vigl B, Brown LF, Detmar M. VEGF-A produced by chronically inflamed tissue induces lymphangiogenesis in draining lymph nodes. Blood. 2007;110(9):3158-3167.

46. Liao S, Ruddle NH. Synchrony of high endothelial venules and lymphatic vessels revealed by immunization. J Immunol. 2006;177(5):3369-3379.

47. Mumprecht V, Roudnicky F, Detmar M. Inflammation-induced lymph node lymphangiogenesis is reversible. Am J Pathol. 2011;180(3):874-879.

48. Tan KW, et al. Expansion of cortical and medullary sinuses restrains lymph node hypertrophy during prolonged inflammation. J Immunol. 2012;188(8):4065-4080.

49. Yao LC, Baluk P, Feng J, McDonald DM. Steroid-resistant lymphatic remodeling in chronically inflamed mouse airways. Am J Pathol. 2010;176(3):1525-1541.

50. Kelley PM, Connor AL, Tempero RM. Lymphatic vessel memory stimulated by recurrent inflammation. Am J Pathol. 2013;182(6):2418-2428.

51. Tewalt EF, Cohen JN, Rouhani SJ, Engelhard VH. Lymphatic endothelial cells - key players in regulation of tolerance and immunity. Front Immunol. 2012;3:305

52. Dietrich T, et al. Cutting edge: lymphatic vessels, not blood vessels, primarily mediate immune rejections after transplantation. J Immunol. 2010; 184(2):535-539.

53. Baluk P, et al. Functionally specialized junctions between endothelial cells of lymphatic vessels. J Exp Med. 2007;204(10):2349-2362.

54. Pflicke H, Sixt M. Preformed portals facilitate dendritic cell entry into afferent lymphatic vessels. J Exp Med. 2009;206(13):2925-2935.

55. Serhan CN, Savill J. Resolution of inflammation: the beginning programs the end. Nat Immunol. 2005;6(12):1191-1197.

56. Ji RC. Macrophages are important mediators of either tumor- or inflammation-induced lymphangiogenesis. Cell Mol Life Sci. 2012;69(6):897-914.

57. Schoppmann SF, et al. Tumor-associated macrophages express lymphatic endothelial growth factors and are related to peritumoral lymphangiogenesis. Am J Pathol. 2002;161(3):947-956.

58. Forster R, Davalos-Misslitz AC, Rot A. CCR7 and its ligands: balancing immunity and tolerance. Nat Rev Immunol. 2008;8(5):362-371.

59. Tal O, et al. DC mobilization from the skin requires docking to immobilized CCL21 on lymphatic endothelium and intralymphatic crawling. J Exp Med. 2011;208(10):2141-2153.

60. Johnson LA, Clasper S, Holt AP, Lalor PF, Baban D, Jackson DG. An inflammation-induced mechanism for leukocyte transmigration across lymphatic vessel endothelium. J Exp Med. 2006;203(12):2763-2777.

61. Angeli V, Randolph GJ. Inflammation, lymphatic function, and dendritic cell migration. Lymphat Res Biol. 2006;4(4):217-228.

62. Chen L, et al. Vascular endothelial growth factor receptor-3 mediates induction of corneal alloimmunity. Nat Med. 2004;10(8):813-815.

63. Nykanen AI, et al. Targeting lymphatic vessel activation and CCL21 production by vascular endothelial growth factor receptor-3 inhibition has novel immunomodulatory and antiarteriosclerotic effects in cardiac allografts. Circulation. 2010;121(12):1413-1422.

64. El-Chemaly S, Pacheco-Rodriguez G, Ikeda Y, Malide D, Moss J. Lymphatics in idiopathic pulmonary fibrosis: new insights into an old disease. Lymphat Res Biol. 2009;7(4):197-203.

65. Marchal-Somme J, et al. Cutting edge: nonproliferating mature immune cells form a novel type of organized lymphoid structure in idiopathic pulmonary fibrosis. J Immunol. 2006;176(10):5735-5739.

66. Pierce EM, et al. Idiopathic pulmonary fibrosis fibroblasts migrate and proliferate to CC chemokine ligand 21. Eur Respir J. 2007;29(6):1082-1093.

67. Jurisic G, Sundberg JP, Detmar M. Blockade of VEGF receptor-3 aggravates inflammatory bowel disease and lymphatic vessel enlargement. Inflamm Bowel Dis. 2013;19(9):1983-1989.

68. Ganta VC, et al. Angiopoietin-2 in experimental colitis. Inflamm Bowel Dis. 2010;16(6):1029-1039.

69. Jang JY, et al. Conditional ablation of LYVE-1+ cells unveils defensive roles of lymphatic vessels in intestine and lymph node. Blood. 2013;122(13):2151-2161.

70. Fogt F, Pascha TL, Zhang PJ, Gausas RE, Rahemtulla A, Zimmerman RL. Proliferation of D2-40-expressing intestinal lymphatic vessels in the lamina propria in inflammatory bowel disease. Int J Mol Med. 2004;13(2):211-214.

71. Heatley RV, Bolton PM, Hughes LE, Owen EW. Mesenteric lymphatic obstruction in Crohn's disease. Digestion. 1980;20(5):307-313.

72. von der Weid PY, Rehal S, Ferraz JG. Role of the lymphatic system in the pathogenesis of Crohn's disease. Curr Opin Gastroenterol. 2011;27(4):335-341.

73. Sura R, Colombel JF, Van Kruiningen HJ. Lymphatics, tertiary lymphoid organs and the granulomas of Crohn's disease: an immunohistochemical study. Aliment Pharmacol Ther. 2011;33(8):930-939.

74. Pedica F, Ligorio C, Tonelli P, Bartolini S, Baccarini P. Lymphangiogenesis in Crohn's disease: an immunohistochemical study using monoclonal antibody D2-40. Virchows Arch. 2008;452(1):57-63.

75. Geleff S, Schoppmann SF, Oberhuber G. Increase in podoplanin-expressing intestinal lymphatic vessels in inflammatory bowel disease. Virchows Arch. 2003;442(3):231-237.

76. Rahier JF, et al. Decreased lymphatic vessel density is associated with postoperative endoscopic recurrence in Crohn's disease. Inflamm Bowel Dis. 2013;19(10):2084-2090.

77. Alexander JS, Chaitanya GV, Grisham MB, Boktor M. Emerging roles of lymphatics in inflammatory bowel disease. Ann N Y Acad Sci. 2010; 1207(suppl 1):E75-E85.

78. Donath MY, Shoelson SE. Type 2 diabetes as an inflammatory disease. Nat Rev Immunol. 2011; 11(2):98-107.

79. Shoelson SE, Lee J, Goldfine AB. Inflammation and insulin resistance. J Clin Invest. 2006; 116(7):1793-1801.

80. Yin $\mathrm{N}$, et al. Lymphangiogenesis is required for pancreatic islet inflammation and diabetes. PLoS One. 2011;6(11):e28023

81. Moriguchi P, Sannomiya P, Lara PF, Oliveira-Filho RM, Greco KV, Sudo-Hayashi LS. Lymphatic system changes in diabetes mellitus: role of insulin and hyperglycemia. Diabetes Metab Res Rev. 2005;21(2):150-157. 
82. Haemmerle M, et al. Enhanced lymph vessel density, remodelling and inflammation is reflected by gene expression signatures in dermal lymphatic endothelial cells in type 2 diabetes. Diabetes. 2013;62(7):2509-2529.

83. Yin N, Zhang N, Xu J, Shi Q, Ding Y, Bromberg JS. Targeting lymphangiogenesis after islet transplantation prolongs islet allograft survival. Transplantation. 2011;92(1):25-30.

84. Cursiefen C, et al. Inhibition of hemangiogenesis and lymphangiogenesis after normal-risk corneal transplantation by neutralizing VEGF promotes graft survival. Invest Ophthalmol Vis Sci. 2004;45(8):2666-2673

85. Nakao S, Hafezi-Moghadam A, Ishibashi T. Lymphatics and lymphangiogenesis in the eye. $J O p h$ thalmol. 2012;2012:783163.

86. Bock F, et al. Novel anti(lymph)angiogenic treatment strategies for corneal and ocular surface diseases. Prog Retin Eye Res. 2013;34:89-124.

87. Oh JY, Ko JH, Lee HJ, Kim MK, Lee JH, Wee WR The antigenicity of ex vivo cultivated human corneal limbal epithelial and stromal cells: temporal changes in vitro. Cornea. 2010;29(11):1302-1307.
88. Zakaria N, Van Marck V, Koppen C, Berneman Z, Tassignon MJ. Lymphangiogenesis may play a role in cultivated limbal stem cell transplant rejection. Ocul Immunol Inflamm. 2012;20(5):381-383.

89. Cursiefen C, Maruyama K, Jackson DG, Streilein JW, Kruse FE. Time course of angiogenesis and lymphangiogenesis after brief corneal inflammation. Cornea. 2006;25(4):443-447.

90. Kerjaschki D, et al. Lymphatic neoangiogenesis in human kidney transplants is associated with immunologically active lymphocytic infiltrates. J Am Soc Nephrol. 2004;15(3):603-612. 